\title{
Sedative effects of peanut (Arachis hypogaea L.) leaf aqueous extracts on brain ATP, AMP, Adenosine and Glutamate/GABA of rats
}

\author{
Xiao-Yan Zu, Zhen-Ya Zhang*, Ji-Qiang Liu, Hong-Hai Hu, Guo-Qing Xing, Ying Zhang, Di Guan
}

Graduate School of Life and Environmental Sciences, Tsukuba University, Tsukuba, Japan; *Corresponding author.

Email: tyou6688@sakura.cc.tsukuba.ac.jp

Received 26 December 2009; revised 10 January 2010; accepted 17 January 2010.

\section{ABSTRACT}

Peanut (Arachis hypogaea L.) leaf aqueous extracts (PLAE) has been reputed to be a type of sleep-aid in China. To investigate the sedative effects and effect pathways of PLAE, rats $(n=31)$ were employed in two experiments and intragastrically administrated of (1) distilled water, PLAE $(500 \mathrm{mg} / \mathrm{kg}$ body weight $(B W)$ ) and peanut stem aqueous extracts (PSAE, 500 $\mathrm{mg} / \mathrm{kg} \mathrm{BW}$ ); (2) 0, 100 or $500 \mathrm{mg} / \mathrm{kg}$ BW of PLAE, respectively for at least 14 days. Six relevant neurotransmitters were measured finally. Experiment-1 ( $n$ $=16$ ) results showed that the brain Lactate were significantly elevated $(p<0.05)$ in rat cerebrums after PLAE administrations, compared with Control and PSAE groups. In respect of brain energy system, significant degradations of the brain adenosine triphosphate (ATP) $(p<0.05)$ were observed in the brainstems and even the whole brains of rats though PLAE treatments. Moreover, we found that the brain Adenosine monophosphate (AMP) were clearly decreased $(p<0.05)$ in rat cerebrum and brainstem regions, while the brain Adenosine revealed an increasing propensity $(p=0.076)$ in the cerebrums of freely behaving rats. After experiment-2 $(n=15)$, the $\gamma$-aminobutyric acid (GABA) concentrations were statistically $(p<0.05)$ enhanced and the ratios of Glutamate/GABA were simultaneously reduced $(p<$ $0.05)$ in rat brainstems, no matter which one dose $(100$ or $500 \mathrm{mg} / \mathrm{kg} \mathrm{BW}$ ) of PLAE were used. Results indicated that PLAE could influence the target neurotransmitters that related to rat circadian rhythms in the specific brain regions, possessing the potentialities as a sedative or sleep-aid for hypnic therapy purposes.

Keywords: Arachis Hypogaea L. Leaf Aqueous Extracts; Sedative Effects; Rats; Neurotransmitters

\section{INTRODUCTION}

Given the high prevalence of insomnia and hypnotics addiction worldwide, herb sedatives have attracted increasing research interests in terms of substituting the drug-addictive hypnotics. However, to our best knowledge, most of herb-sedative studies remain inconclusive in support of their effectiveness [1]. Therefore, a novel herb medicine that is demonstratively available to alleviate insomnia or sleep disorders is highly desired nowadays.

Peanut (Arachis hypogaea L.) leaf aqueous extracts (PLAE) have received a long reputation in china as an abirritative remedy to ease various sleep disorders [2], and clinically validated by modernistic medical approaches $[3,4]$. However, many those researches only focus on the clinical effects, and relevant studies on their deep effect mechanisms are still lacking. Our studies therefore carried out two rat experiments which lied in clarifying PLAE effects and pathways in the sleep regulation as well as evaluating their efficacies on spontaneous circadian rhythms of freely behaving rats.

By understanding how the animal circadian rhythms are influenced via brain nerve system and neurotransmitters $[5,6]$, in our researches, PLAE efficacy can be identified though brain neurotransmitter variation and thereby we expect that its effect mechanisms can be revealed to some extent though this way. Thus, after intragastric (i.g.) drug administrations we separated rat brain tissues and then measured six neurotransmitters of Lactate, Adenosine triphosphate (ATP), Adenosine monophosphate (AMP), Adenosine (Ad), $\gamma$-aminobutyric acid (GABA), and Glutamate (Glu) using HPLC (High performance liquid chromatography) or Auto Amino Acid Analyzer, to evaluate the hypnotic effects of PLAE and further elucidate their effect roles correlated to PLAE on rat sleep modulation.

\section{MATERIALS AND METHODS}

\subsection{Animals, Plant Extracts and Reagents}

Male Sprague-Dawley rats ( 8 weeks of age, weight of $270 \pm 30 \mathrm{~g}$ ) were used as research animals. They were 
housed at ambient circumstance of $25^{\circ} \mathrm{C}$ with $12 \mathrm{~h}$ light/dark cycles (light on 08:00, light off 20:00), and approached food and water ad libitum. All rat experiments were carried out in a humane manner after receiving approval from Institutional Animal Experiment Committee of the Tsukuba University (Japan), and in accordance with the regulations for Animal Experiments and fundamental guidelines under the jurisdiction of the Japanese Ministry of Education, Culture, Sports, Science and Technology. The PLAE and peanut stem aqueous extracts (PSAE) were extracted respectively from $98{ }^{\circ} \mathrm{C}$ water ( $3 \mathrm{~h}$, twice), following a filtration to remove the residues. Their brown powders were obtained though rotary evaporation and freeze drying. The regents of ATP, AMP and Ad were purchased from Sigma Chemical Co. (St. Louis, MO, USA). Other chemicals used were purchased from Wako Pure Chemical Industries, Ltd. (Japan).

\subsection{Experimental Protocols}

Experiment-1: Sixteen rats were habituated in an animal lab for at least 7 days, then randomly divided into the following groups: Control ( $n=5$, distilled water), PLAE $(n=6)$ and PSAE $(n=5)$. As described before [7], all drug administrations were conducted intragastrically (i.g.) for 14 days in a dose of $500 \mathrm{mg} / \mathrm{kg}$ body weight (BW) before 8:00 (prior to the beginning of the light phase). Experiment-2: After habituated for 7 days, the rats $(n=15)$ employed were equally divided into three groups, fed with 0,100 or $500 \mathrm{mg} / \mathrm{kg} \mathrm{BW}$ of PLAE (i.g.) respectively for at least 14 days. After trials, the rats were anaesthetized by Urethane (intra-peritoneal (i.p.) injection, $200 \mathrm{mg} / \mathrm{ml}, 0.5 \mathrm{ml} / 100 \mathrm{~g} \mathrm{BW}$ ), and dissected rapidly to separate their whole brains. To verify the neurotransmitter variation in diverse brain regions, the whole brain was separated into three parts of cerebrum region, brainstem region (midline involving thalamus and hypothalamus) and cerebellum region. Considering the brain responses to nucleoside and nucleotide synthesis, we froze those brain tissues immediately and stored them at $-80^{\circ} \mathrm{C}$ till the target neurotransmitters were analyzed.

\subsection{Neurotransmitter Analysis}

After experiment-1, samples were firstly thawed and homogenized with $4{ }^{\circ} \mathrm{C}$ saturated trichloroacetic acid (TCA) in the sample volume of $25 \%(\mathrm{v} / \mathrm{v})$, followed a centrifugation to remove the protein sediments. The $\mathrm{pH}$ values in cooled supernatants were then neutralized to $5-6$ by $\mathrm{NaOH}$. After filtered $(0.45 \mu \mathrm{m}$ membrane), the supernatants were analyzed by the HPLC (Japanese Jasco International Co., Ltd). Brain Lactates were measured though RI detector of HPLC on the basis of the methods described before (Hallstrom et al., 1989), and $0.1 \% \mathrm{H}_{3} \mathrm{PO}_{4}$ were used as the mobile phase. Analyzing brain neurotransmitters of ATP, AMP and Ad, were per- formed according to the methods reported previously [8] with minor modifications. Simply, the samples were analyzed by the HPLC with Capcell-Pak $\mathrm{C}_{18}$ column $(4.6 \mathrm{~mm}$ I.D. $\times 150 \mathrm{~mm}$, particle size of $5 \mu \mathrm{m})$ in a flow rate of $1 \mathrm{ml} / \mathrm{min}$ at the detection wavelength of $254 \mathrm{~nm}$. During testing, the mobile phase was $0.01 \mathrm{~mol} / \mathrm{L}$ phosphate buffer solution (PBS, $\mathrm{pH}$ 6.5) that mixed with methanol $(99.7 \%)$ in a ratio of $85 / 15(\mathrm{v} / \mathrm{v})$. Concentrations of ATP and its metabolites were calculated by comparing peak areas with appropriate standards.

After experiment-2, the brain samples homogenized were mixed with sulfosalicylic acid (3\%) for $15 \mathrm{~min}$ prior to centrifugation. The $\mathrm{pH}$ value of supernatants were then adjusted to $2-3$ using $\mathrm{LiOH}(3 \mathrm{~mol} / \mathrm{L})$. Brain GABA and Glu in samples were determined by an auto amino acid analyzer (JLC-500/V2, Jeol Ltd., Tokyo, Japan) in accordance with the manufacturer's specifications. Briefly, it was an ionic exchange chromatography with a stepwise elution of free amino acids, and detection with ninhydrine. The final results were calculated on the basis of the corresponding standards, and expressed as concentrations of nmol/mg in brain tissues.

\subsection{Statistical Analysis}

The obtained data were analyzed using a two-tailed Student's $t$-test, and results were expressed as mean \pm SD. Statistic difference was considered to be significant at $p<0.05\left(^{*}\right)$.

\section{RESULTS}

\subsection{Brain ATP}

As results shown in Figure 1, the ATP of control (Con.) group in the cerebrum, brainstem, and whole brain were $0.114 \pm 0.020,0.109 \pm 0.015$ and $0.134 \pm 0.022 \mathrm{nmol} / \mathrm{mg}$, respectively. Contrasted to Control (Con.) Groups, the brain ATP declined in both of the whole brain and the single brain region after PLAE administrations. In detail, The ATP from PLAE groups were slightly reduced in cerebrums, while significant degradations were observed $(p<0.05)$ in the brainstems and whole brains on comparison with Con. groups. In the case of PSAE treatments, the enhancements of ATP were occurred in all brain regions, especially in the cerebrums.

\subsection{Brain AMP}

In experiment-1, we also obtained the AMP concentrations of $0.072 \pm 0.013,0.078 \pm 0.005,0.082 \pm 0.007$ $\mathrm{nmol} / \mathrm{mg}$ respectively in the cerebrum, brainstem, and whole brain from Con. groups. As can been seen from Figure 2, after PLAE treated, the AMP were decreased in almost all brain regions (cerebellum results were not showed in this figure), particularly reduced $(p<0.05)$ in the cerebrum and brainstem regions. The AMP from PSAE groups showed no statistic changes compared 


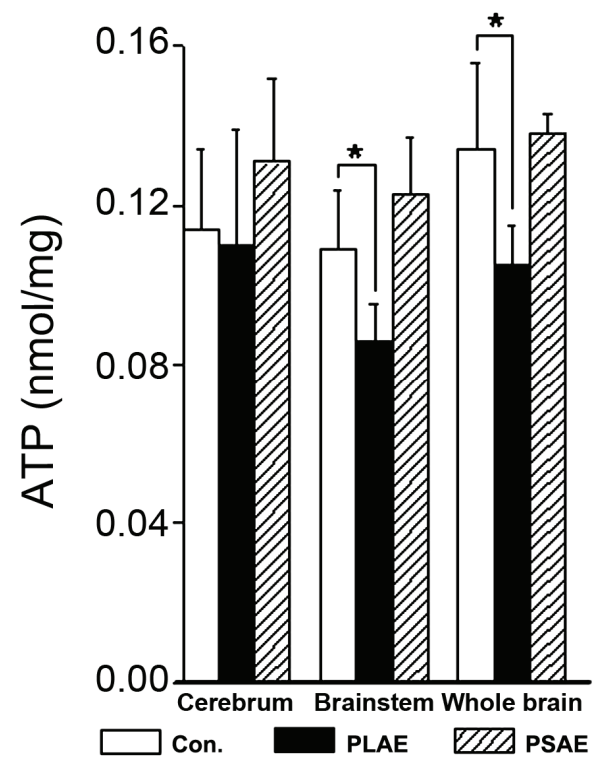

Figure 1. ATP concentrations in the cerebrum, brainstem and whole brain of freely behaving rats at experiment-1. Open bars, Con. (Control, distilled water); closed bars, PLAE (500 mg/kg BW); diagonal bars, PSAE $(500 \mathrm{mg} / \mathrm{kg} \mathrm{BW})$. Data were expressed as mean \pm SD of 5-6 independent samples $\left({ }^{*} p<0.05\right.$, vs. Con.).

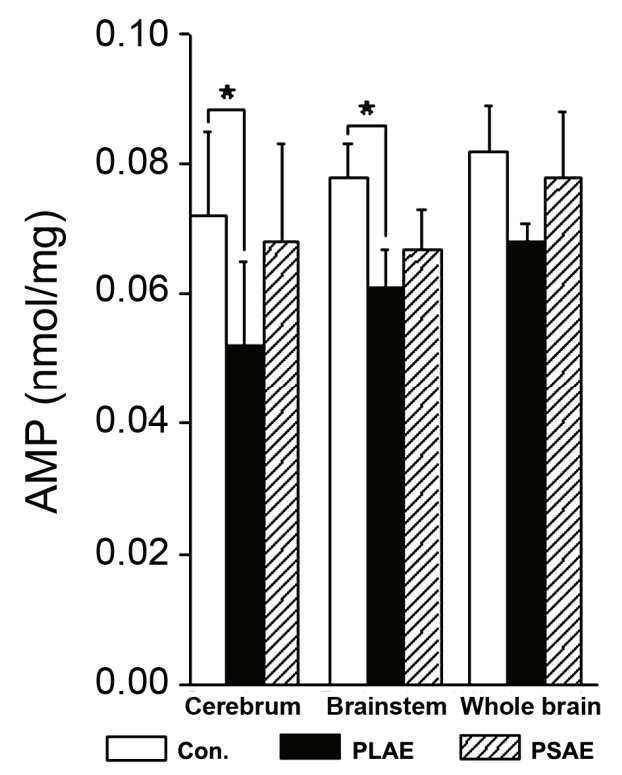

Figure 2. AMP concentrations in the cerebrum, brainstem and whole brain of freely behaving rats at experiment-1. Open bars, Con. (Control, distilled water); closed bars, PLAE (500 mg/kg BW); diagonal bars, PSAE $(500 \mathrm{mg} / \mathrm{kg} \mathrm{BW})$. Data were expressed as mean \pm SD of 5-6 independent samples $\left({ }^{*} p<0.05\right.$, vs. Con.). with group Con. though the trails, although the declines were observed as well.

\subsection{Brain Ad and Lactate}

Figure 3 showed the results of Ad and lactate in Cerebrum, and revealed that the Lactate changes were consistent with Ad in cerebrum after PLAE administrations in Experiment-1. As can be seen, compared with group Con. and PSAE, the Ad amelioration $(p=0.076)$ in PLAE group was occurred in cerebrum where played the main roles on modulating animal sleepiness. And the Lactate was elevated $(p<0.05)$ in cerebrum simultaneously after PLAE treated. On the other hand, PSAE results presented only slight differences in contrast to the Con. groups.

\subsection{Brain GABA}

Brain GABA of PLAE-0 group were $0.767 \pm 0.180$ and $0.767 \pm 0.079 \mathrm{nmol} / \mathrm{mg}$ respectively in the brainstem and Whole brain. And the GABA results of cerebrums and cerebellums were monotonous and eliminated from Figure 4. In the results, we found that the GABA concentrations exceeded statistically $(p<0.05)$ than PLAE- 0 group in brainstems at both PLAE-100 and PLAE-500 groups. The GABA from PLAE-500 group showed significant improvement $(p<0.05)$ even in the whole brain of the experimental rats.

\subsection{Brain Glu}

After experiment-2, we investigated the brain Glu contents in different brain regions simultaneously. As Figure 5

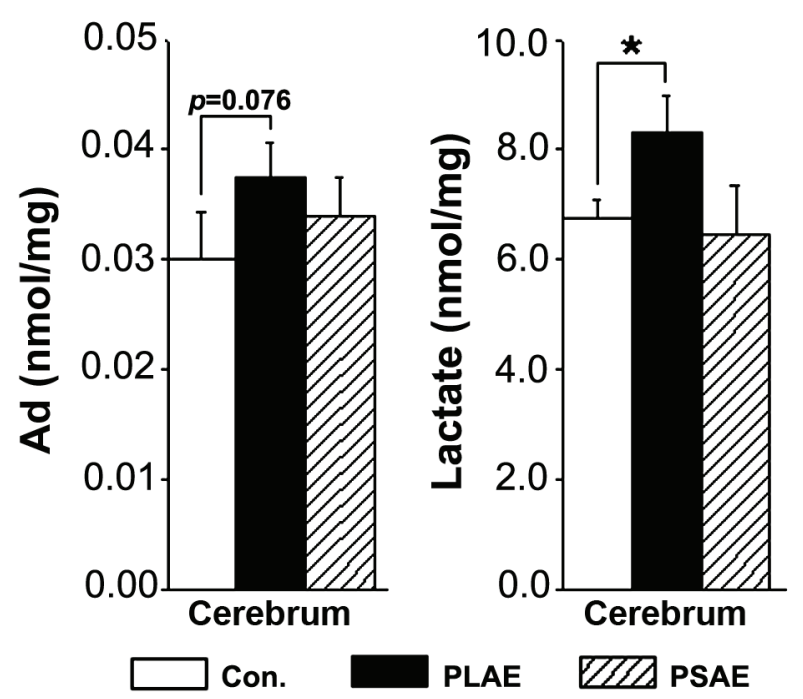

Figure 3. Concentrations of Ad and Lactate in the cerebrum of freely behaving rats at experiment-1. Open bars, Con. (Control, distilled water); closed bars, PLAE (500 mg/kg BW); diagonal bars, PSAE (500 mg/kg BW). Data were expressed as mean \pm SD of 5-6 independent samples ( ${ }^{*} p<0.05$, vs. Con.). 


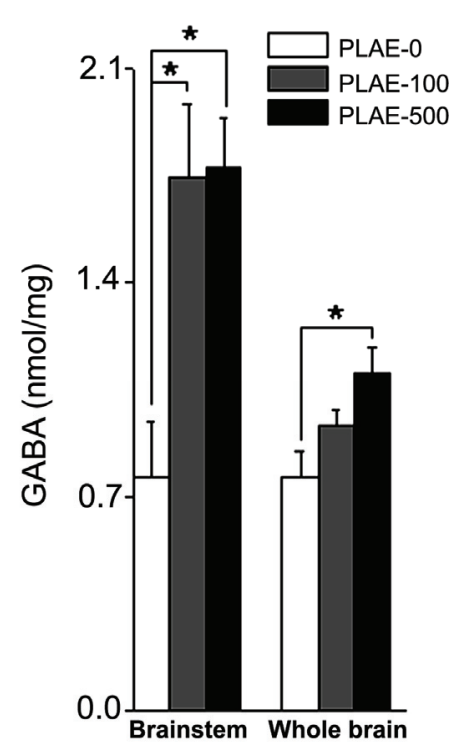

Figure 4. GABA concentrations in the brainstem and whole brain of freely behaving rats at experiment-2. Open bars, PLAE-0 (Control, without PLAE); gray bars, PLAE-100 (100 mg/kg BW of PLAE); closed bars, PLAE-500 (500 mg/kg BW of PLAE). Data were expressed as mean \pm SD of five independent samples $\left({ }^{*} p<0.05\right.$, vs. PLAE-0).

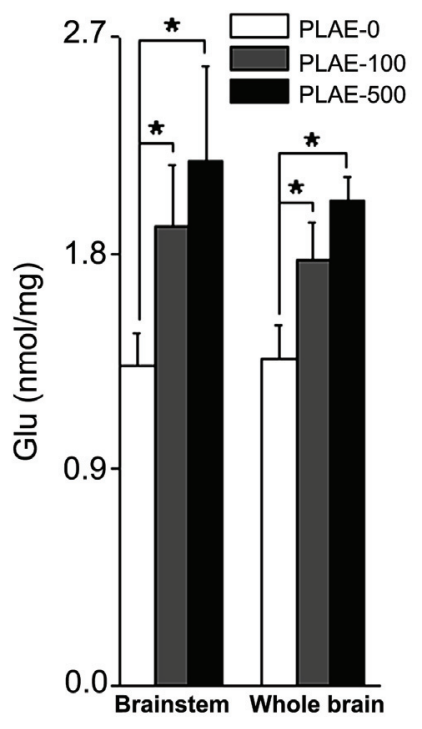

Figure 5. Glu concentrations in the brainstem and whole brain of freely behaving rats at experiment-2. Open bars, PLAE-0 (Control, without PLAE); gray bars, PLAE-100 (100 mg/kg BW of PLAE); closed bars, PLAE-500 (500 mg/kg BW of PLAE). Data were expressed as mean \pm SD of five independent samples $\left({ }^{*} p<0.05\right.$, vs. PLAE-0). shown, the Brain Glu in the brainstem and whole brain were $1.330 \pm 0.139$ and $1.361 \pm 0.141 \mathrm{nmol} / \mathrm{mg}$ respectively, which elucidated that the rats in PLAE-0 group possessed the higher contents of initial brain Glu than the brain GABA. Moreover, on comparison with PLAE-0 groups, the Glu concentrations were greatly raised $(p<0.05)$ in the brainstem as well as the whole brain, no matter which one dosage (100 or $500 \mathrm{mg} / \mathrm{kg}$ BW) of PLAE were administered in experiment-2.

\subsection{The ratio of Glu/GABA}

Considering the results in Figure 4 and 5, the ratios of Glu/GABA was important to evaluate the excitatory or suppressive state of the rat brain, we thereby calculated it and presented the results in Figure 6. Available variances have not been observed in the whole brains of the three groups. Moreover, compared to the PLAE-0 groups, the ratio of Glu/GABA in PLAE-100 and PLAE500 groups were specifically decreased $(p<0.05)$ in brainstems where showed important relations with the two neurotransmitters.

\section{DISCUSSION}

\subsection{Effects of PLAE and PSAE on Brain Energy Metabolism}

ATP is the direct energy source of life activities. Its decline (Figure 1) in rat brain on our investigations re flected

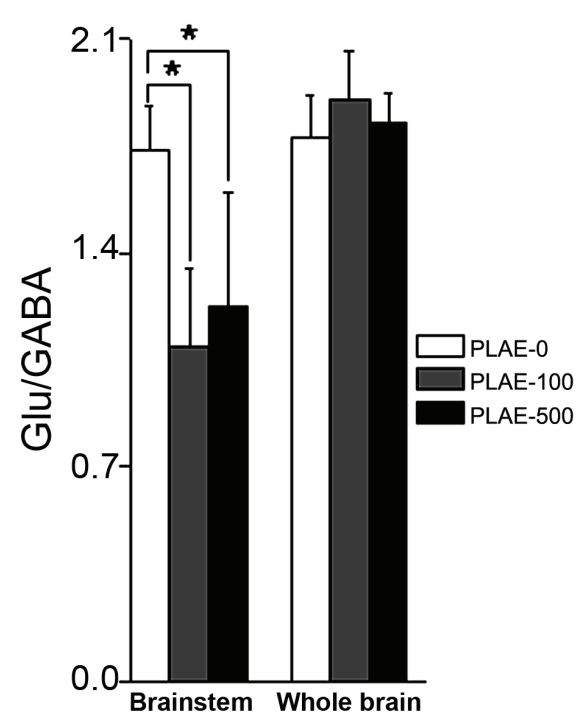

Figure 6. Ratios of Glu/GABA in the brainstem and whole brain of freely behaving rats at experiment-2. Open bars, PLAE-0 (Control, without PLAE); gray bars, PLAE-100 (100 $\mathrm{mg} / \mathrm{kg}$ BW of PLAE); closed bars, PLAE-500 $(500 \mathrm{mg} / \mathrm{kg}$ BW of PLAE). Data were expressed as mean $\pm \mathrm{SD}$ of five independent samples $(* p<0.05$, vs. PLAE- 0 ). 
the requirement of energy reserves, and indicated the potential of rat sleepiness. AMP can transfer into ATP or degraded into Ad [9]. According to the deficiency of ATP in the results, the reduction of AMP (Figure 2) in group PLAE elucidated the tendency of its breaking down to Ad. As reported previously, Ad is a critical neurotransmitter in brain sleep system [10], which accumulates in the cholinergic basal forebrain of cerebrum, has been proposed as one of the important homeostatic sleep factors [11]. In our researches, as a result of the deficiency of high-energy phosphates, the observed increases of Ad concentrations in cerebrums reflected a bio-energetic stress of animals. Energy depletion can build up the sleep pressure [7] and it is an available pathway and measure to explain the PLAE induced sleep propensity. From experiment-1, the great decrease of the ATP and AMP strongly indicated that the PLAE was promising in driving homeostatic sleeps of freely behaving rats. Besides, the insignificant accumulation of Ad in the PLAE group suggested a mild effect of PLAE on sleep ameliorating.

Lactate, an end product of metabolic system, can be regarded as an additional metabolic marker of the sleep pressure. Our results (Figure 3) illuminated that Lactate changed together with $\mathrm{Ad}$, in agreement with those reported previously [12] and indicative of the PLAE contributions in sedative or hypnic propensities.

In all PSAE investigations, the rat brain energy system presented the great enhancement of ATP in cerebrum compared with Control and PLAE groups. However, such variations were disadvantageous for establishing the sleep pressure and meliorating the sleep of our experimental rats.

\subsection{Effects of PLAE on Brain GABA, Glu and the Ratio of Glu/GABA}

GABA is a type of inhibitory neurotransmitter amino acid in brain. To clarify the PLAE effects on brain GABA, fifteen rats were employed in experiment- 2 and randomly divided into three groups, and treated with PLAE in diverse dosages for 14 days. The GABAcontained neurons in brainstem and thalamic nucleus released GABA neurotransmitters [13,14], which can response for inhibiting target neurons of the arousal systems in brainstem [15] and play an important role in meliorating rats' sleep [6]. Thus, the GABA increase in our studies (Figure 4) not only suggested the specific effect region (brainstem) of GABA-neurotransmitter, and also testified the sedative efficacy of PLAE.

It is well demonstrated that Glu play an excitatory role in animal brain, and then drives the animals to arousal. Glu receptors, expressed in the basal forebrain [16,17], stimulate gamma and theta electroencephalographic (EEG) activities of arousal and then suppress rat sleepiness $[18,19]$. In these researches, we found that PLAE also aroused the neurons to release Glu neurotransmitters (Figure 5) for exciting the rat brains.

In deed, GABA can be formed from Glu primarily via the action of Glu decarboxylase [20]. Glu/GABA in brainstem is a type of available parameters that can characterize the state of sleep-wake system [21]. Since GABA is inhibitory and Glu is excitatory, both of them control many tranquilizing or exciting processes in rat brain. Apparently, PLAE we used stimulated both the brain Glu and the brain GABA. However, the sleep-modulation effects of Glu sited specifically in perifornical-lateral hypothalamic area [22] of the brainstem which is the main region that releases GABA to inhibit the arousal of animal. Hence, the Glu/GABA decrease in this area (Figure 6) indicated superior GABA efficacy than Glu, and preferred to drive rats into drowsiness. On the other hand, as a mild tranquilizer, it was not surprising that PLAE significantly elevated GABA-mediated neurotransmission, and reduced Glu/ GABA in target brain region. Thus, in profile, it was still suggested the PLAE efficacy on spontaneous sleep improvement.

In summary, PLAE resulted in the great ATP consumption, slight Ad accumulation, as well as the significant decrease of Glu/GABA in corresponding brain areas. It revealed a mild hypnotic effect of PLAE on sleep ameliorations. In the case of sedatives, the PSAE was considered to be ineffective on meliorating rats' sleeps at least view the matter from our investigations. On the other hand, in these researches, more detailed works on the effect components of such PLAE are desired to be determined in the future. Moreover, apart from the aqueous extracts of peanut leaves and stems, alcohol extracts of them are needed as well to as the contrasts in further investigations.

\section{REFERENCES}

[1] Diana, M.T., Carol, A.L., Heidi, P. and Michael, V.V. (2007) A systematic review of valerian as a sleep aid: Safe but not effective. Sleep Medicine Reviews, 11, 209-230.

[2] Wang, Q.C., Xu, J. and Shi, M. (2001) Clinical efficacy of Groundnut leaves on insomnia treatments. Shanghai Journal of Traditional Chinese Medicine, 5, 8-10.

[3] Hu, P.F., Fan, R.P., Li, Y.P. and Pang, C.Y. (2001) Studies on pharmacological action of Luohuashengzhiye extracts. Chinese Traditional Patent Medicine, 23, 919920 .

[4] Wang, Y.F., Li, H.F., Xu, Y.F., Zhang, Y.L., Xu, D.S., Xiao, L.M. and Li, X.M. (2001). Clinical confirmation of preparation from the branch and leaf of peanut in treating insomnia. Shanghai Journal of Traditional Chinese Medicine, 35, 8-10.

[5] Saper, C.B., Scammell, T.E. and Lu, J. (2005) Hypothalamic regulation of sleep and circadian rhythms. Nature, 437, 1257-1263. 
[6] Jones, B.E. (2005) From waking to sleeping: Neuronal and chemical substrates. Trends in Pharmacological Sciences, 26, 578-586.

[7] Thakkar, M.M., Engemann, S.C., Walsh, K.M. and Sahota, P.K. (2008) Adenosine and the homeostatic control of sleep: Effects of A1 receptor blockade in the perifornical lateral hypothalamus on sleep-wakefulness. Neuroscience, 153, 875-880.

[8] Schweinsberg, P.D. and Loo, T.L. (1980) Simultaneous analysis of ATP, ADP, AMP, and other purines in human erythrocytes by high-performance liquid chromatography. Journal of Chromatography, 181, 103-107.

[9] Dworak, M., Diel, P., Voss, S., Hollmann, W.K. and Strüder, H. (2007) Intense exercise increases adenosine concentrations in rat brain: Implications for a homeostatic sleep drive. Neuroscience, 150, 789-795.

[10] Huang, Z.L., Urade, Y. and Hayaishi, O. (2007) Prostaglandins and adenosine in the regulation of sleep and wakefulness. Current Opinion in Pharmacology, 7, 33-38.

[11] Kalinchuk, A.V., Urrila, A.S., Alanko, L., Heiskanen, S., Wigren, H.K., Suomela, M., Stenberg, D. and PorkkaHeiskanen, T. (2003) Local energy depletion in the basal forebrain increases sleep. European Journal of Neuroscience, 17, 863-869.

[12] Wigren, H.K., Schepens, M., Matto, V., Stenberg, D. and Porkka-Heiskanen, T. (2007) Glutamatergic stimulation of the basal forebrain elevates extracellular adenosine and increases the subsequent sleep. Neuroscience, 147, 811-823.

[13] Maloney, K.J., Mainville, L. and Jones, B.E. (1999) Differential c-Fos expression in cholinergic, mono-aminergic and GABAergic cell groups of the pontomesencephalic tegmentum after paradoxical sleep deprivation and recovery. Neuroscience, 19, 3057-3072.

[14] Maloney, K.J., Mainville, L. and Jones, B.E. (2000) c-Fos expression in GABAergic, serotonergic and other neurons of the pontomedullary reticular formation and raphe after paradoxical sleep deprivation and recovery. Neuroscience, 20, 4669-4679.

[15] Sherin, J.E., Shiromani, P.J., McCarley, R.W. and Saper, C. B. (1996) Activation of ventrolateral preoptic neurons during sleep. Science, 271, 216-219.

[16] Martin, L.J., Blackstone, C.D., Levey, A.I., Huganir, R. L. and Price, D.L. (1993) Cellular localizations of AMPA glutamate receptors within the basal forebrain magnocellular complex of rat and monkey. Neuroscience, $\mathbf{1 3}$ 2249-2263.

[17] Page, K.J. and Everitt, B.J. (1995) The distribution of neurons coexpressing immunoreactivity to AMPAsensitive glutamate receptor subtypes (GluR1-4) and nerve growth factor receptor in the rat basal forebrain. European Journal of Neuroscience, 7, 1022-1033.

[18] Cape, E.G. and Jones, B.E. (2000) Effects of glutamate agonist versus procaine microinjections into the basal forebrain cholinergic cell area upon gamma and theta EEG activity and sleep-wake state. European Journal of Neuroscience, 12, 2166-2184.

[19] Fournier, G.N., Materi, L.M., Semba, K. and Rasmusson, D.D. (2004) Cortical acetylcholine release and electroencephalogram activation evoked by ionotropic glutamate receptor agonists in the rat basal forebrain. Neuroscience, 123, 785-792.

[20] Bown, A.W. and Shelp, B.J. (1997) The metabolism and functions of $\gamma$-aminobutyric acid. Plant Physiology, 115, $1-5$.

[21] Schousboe, A., Westergaard, N., Sonnewald, U., Petersen, S.B., Yu, A.C. and Hertz, L. (1992) Regulatory role of astrocytes for neuronal biosynthesis and homeostasis of glutamate and GABA. Progress in Brain Research, 94, 199-211.

[22] Alam, M.A. and Mallick, B.N. (2008) Glutamic acid stimulation of the perifornical-lateral hypothalamic area promotes arousal and inhibits non-REM/REM sleep. Neuroscience Letters, 439, 281-286. 